\title{
Quantification of Cardiovascular Instability in Premature Infants Using Spectral Analysis of Waveforms
}

\author{
S. BIGNALL, P. C. BAILEY, R. P. A. RIVERS, AND T. J. LISSAUER \\ Department of Paediatrics, St. Mary's Hospital Medical School, London, England
}

\begin{abstract}
Spectral analysis was applied to blood pressure and cerebral blood flow velocity recordings in premature infants with respiratory distress in order to quantify respiration-induced cardiovascular variability. Aortic blood pressure was transduced via an umbilical arterial catheter and cerebral blood flow velocity measured in the anterior cerebral artery using a $10 \mathrm{MHz}$ continuous wave Doppler velocimeter in 16 infants $\leqq 32$ wk gestational age. Spectral analysis of the resulting waveforms revealed heart rate and respiratory rate components whose relative amplitudes (heart rate/respiratory rate amplitude ratio) represent an index of that component of variability induced by respiratory events. The mean (heart rate/respiratory rate amplitude) ratio was 47.2 in spontaneously breathing infants and rose to $\mathbf{1 6 5 . 9}$ in infants who were ventilated during muscle paralysis $(p=0.0003)$. Cerebral blood flow velocity recordings showed $R$ components in only 22 of 38 simultaneous recordings. This method can be used to quantify respiration-induced cardiovascular variability and its response to therapy, and may provide a means of identifying infants at risk from brain injury due to an inability to regulate cerebral blood flow. (Pediatr Res 23: 398-401, 1988)
\end{abstract}

\section{Abbreviations}

BP, (aortic) blood pressure

CBFV, cerebral blood flow velocity

$\mathrm{H}$, heart rate frequency component

$R$, respiratory frequency component

$H / R$, ratio of amplitudes of heart rate and respiratory/ frequency components

cv\%, coefficient of variation

Cardiovascular instability has been shown to be associated with an increased risk of brain injury in the sick, premature infant (1). Disorders of the regulation of brain blood flow may allow alterations in cardiac output to manifest in the cerebral circulation (2).

Various forms of cardiovascular instability occur in the early days of life in sick, premature infants. Blood pressure may be low after birth and rises in the first days of life (3); events such as endotracheal suctioning (4), seizures (5), and apneic attacks (6) can cause rapid swings in BP and CBFV, and spontaneous

Received July 31, 1987; accepted December 11, 1987.

Correspondence and reprints Dr. Simon Bignall, Department of Paediatrics, St. Mary's Hospital Medical School, Norfolk Place, London W2 1PG, England.

Supported by the Winnicott Foundation. S.B. was supported by the St. Mary's Hospital Save The Baby Fund. P.C.B, was supported by a research grant from the North West Thames Regional Health Authority. respiration will induce beat to beat fluctuations in these variables. We chose to examine this last form of instability as it is amenable to therapy, e.g. muscle paralysis (7) and as it will be affected by common respiratory conditions and their management.

Short-term fluctuations in BP and CBFV have previously been estimated by calculation of the cv\% over a fixed number of cardiac cycles. Such an estimation does not allow that component of variability which is specifically related to respiration to be identified. The increasing use of muscle paralysis and frequency selective entrainment (8) of spontaneous respiration during mechanical ventilation requires the development of improved methods of measuring the interaction of respiration and the cardiovascular system. Babies who might benefit from these interventions could then be identified and the consequences of therapeutic maneuvers could be evaluated.

Spectral analysis is a mathematical technique that can extract the frequency components of complex waveforms such as aortic blood pressure (9) and estimate the relative contributions of those frequency components to the waveform under study. Using this technique it has been possible to identify several frequency components in aortic BP: $\mathrm{R}(0.5$ to $1.5 \mathrm{~Hz}$ in the newborn) and $\mathrm{H}(1.5$ to $3 \mathrm{~Hz})$ contributions, and low frequency components $(0.01$ to $0.15 \mathrm{~Hz})$ which arise from within the BP and thermoregulatory control systems (10).

We describe a method of identifying and quantifying respiration-induced variations in circulatory waveforms in preterm infants using spectral analysis.

\section{MATERIALS AND METHODS}

The study was approved by the hospital's ethics committee and informed parental consent was obtained. Sixteen premature infants ( $\leqq 32 \mathrm{wk}$ gestational age) undergoing intensive care, including ventilation, for respiratory disorders were studied in the first 2 wk of life. The median gestational age was 27 completed wk (range 24-32) and the median birth weight $969 \mathrm{~g}$ (range 580$2010)$.

Aortic BP was transduced via a saline-filled, 3.5-French gauge, end-hole umbilical arterial catheter at the level of the third lumbar vertebra connected to a quartz pressure transducer (Hewlett Packard 1290A). The natural frequency of the BP system was $10.25 \mathrm{~Hz}$ and the damping coefficient was 0.61 , measured by the rapid flush technique (11). BP and transthoracic impedance signals were amplified and digitized $(128 \mathrm{~Hz}$ sampling frequency, 8-bit conversion) in an Apple $11 \mathrm{e}$ microcomputer (Meadowbank Medical Systems) for later analysis.

CBFV was obtained simultaneously with BP in 12 infants on 38 occasions from the anterior cerebral artery using a $10-\mathrm{MHz}$ continuous wave Doppler velocimeter through the anterior fontanelle. On-line spectral analysis of Doppler shift frequencies was used to ensure good signal quality. A zero-crossing detector 
circuit was used to provide the analogue signal for analysis and this was digitized in the microcomputer simultaneously with the BP signal.

During recordings chest wall movement and the transthoracic impedance waveform were monitored to exclude episodes of crying, agitation, or irregular respiration. All ventilated infants in our unit are sedated with regular doses of pethidine.

The cv\% $(\mathrm{SD} /$ mean $\times 100)$ in aortic $\mathrm{BP}$ was measured in each recording by calculating the area under the $\mathrm{BP}$ curve divided by the duration of each cycle for 20 successive cardiac cycles. Eight$s$ lengths (128 Hz sampling rate, 1024 points) of the BP and CBFV data arrays were subjected to spectral analysis using the Fast Fourier Transform on a PDP-11/73 minicomputer, giving a spectral resolution of $0.125 \mathrm{~Hz}$. No windowing was applied before spectral analysis. The length of signal was chosen to approximate the length of 20 cardiac cycles and to ensure good spectral resolution in the range of interest $(0.5$ to $5 \mathrm{hz})$. The power spectra thus obtained were plotted as frequency on the $x$ axis against the logarithm of amplitude at each frequency on the $y$ axis. The amplitudes of the peaks at the heart rate frequency, $\mathrm{H}$, and at the respiratory rate frequency, $\mathrm{R}$, were then compared. The ratio of the amplitudes of peaks $H$ to $R$ in each recording can then be used to quantify specific respiratory components in aortic BP or CBFV: the lower the value of the $H / R$ ratio, the greater the interference of respiration in the waveform.

In order to test the stability of the $\mathrm{H}$ component 20 consecutive, 8 -s records of aortic BP from the same infant during mixed ventilation (mechanical ventilation and spontaneous respiratory efforts) were analyzed. The cv\% for the amplitude of the $\mathrm{H}$ component was $0.391 \%$, for the $\mathrm{R}$ component $3.093 \%$, and for the $\mathrm{H} / \mathrm{R}$ ratio was $3.05 \%$, i.e. the amplitude of the $\mathrm{H}$ component remains stable despite fluctuations in $\mathrm{BP}$ caused by respiration, and the $H / R$ ratio reflects changes due to respiration alone.

Statistical comparison of the logarithmic transformation of $\mathrm{H} /$ $\mathrm{R}$ ratios in spontaneously breathing infants with those in paralyzed, ventilated infants was performed by mixed model analysis of variance (12). Comparison of $\log \mathrm{cv} \%$ with the $\log \mathrm{H} / \mathrm{R}$ ratio in each $\mathrm{BP}$ recording is represented by the regression coefficient between these variables after allowance for multiple samples from the same baby and the effects of paralysis.

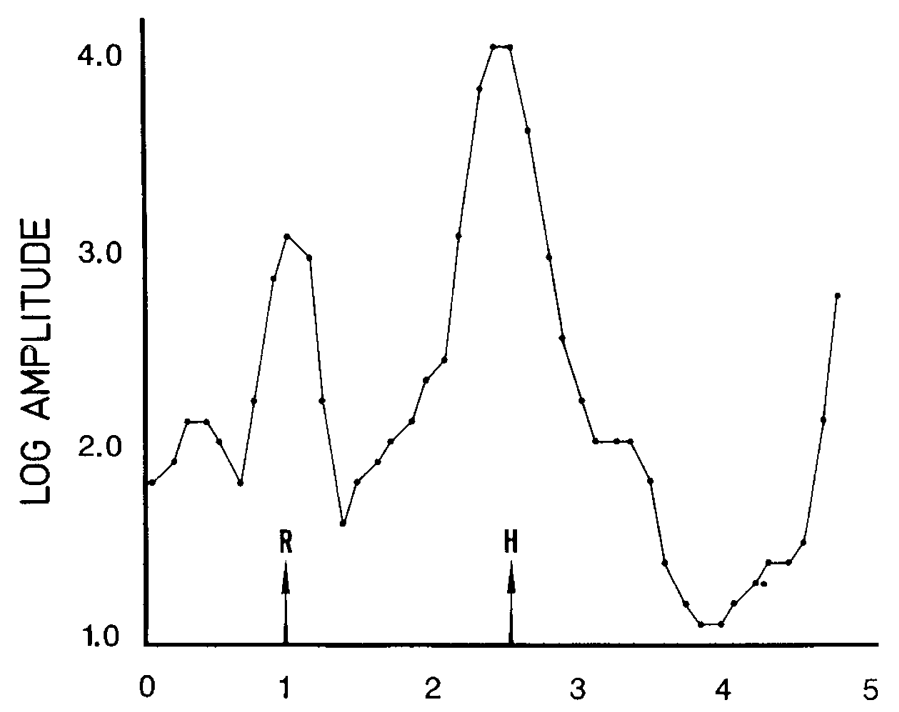

\section{FREQUENCY $(\mathrm{Hz})$}

Fig. 1. Power spectrum of BP during spontaneous respiration. $H$ and $\mathrm{R}$ frequency components are seen around $2-3 \mathrm{~Hz}(120-180$ beats/min) and $1 \mathrm{~Hz}$ (60 breaths/min), respectively. Frequency in $\mathrm{Hz}$ is shown on the $x$ axis. The amplitude at each frequency ( $y$ axis) is shown in arbitrary units on a log scale.

\section{RESULTS}

A typical power spectrum of aortic BP during spontaneous respiration is shown in Figure 1. Amplitude $\mathrm{H}$ is seen at between 2 and $3 \mathrm{~Hz}(120-180$ beats/min) and an R is clearly seen between 0.5 and $1.5 \mathrm{hz}$ (30-90 breaths/min), and is quite distinct from $\mathrm{H}$. A clear R was seen in 112 of $117 \mathrm{BP}$ records analyzed. The mean $\mathrm{H} / \mathrm{R}$ ratio in aortic $\mathrm{BP}$ in spontaneously breathing infants was 47.21 (95\% confidence intervals $254.8,8.75$ ).

Figure 2 shows power spectra for BP (lower panel) and for simultaneously measured CBFV (upper panel) during spontaneous respiratory efforts. The expression of an $\mathrm{R}$ in $\mathrm{BP}$ and $\mathrm{CBFV}$ is clearly seen. In spontaneously breathing infants with a clear $\mathrm{R}$ in aortic $\mathrm{BP}$, simultaneous $\mathrm{CBFV}$ recordings showed a respiratory peak in 22 of 38 records. In paralyzed and ventilated infants the mean amplitude of peak $\mathrm{R}$ at the ventilator cycling rate was low in BP and the $R$ was reduced in CBFV (Fig. 3). The mean $\mathrm{H} / \mathrm{R}$ ratio in aortic $\mathrm{BP}$ in paralyzed and ventilated infants was 165.9 (95\% confidence intervals $895.2,30.74)$. The difference between the $H / R$ ratios in spontaneously breathing and paralyzed infants is statistically significant $(p=0.0003)$.

A comparison of the $H / R$ ratio in 112 recordings of aortic BP with the $\mathrm{cv} \%$ over 20 successive cardiac cycles revealed a regres-
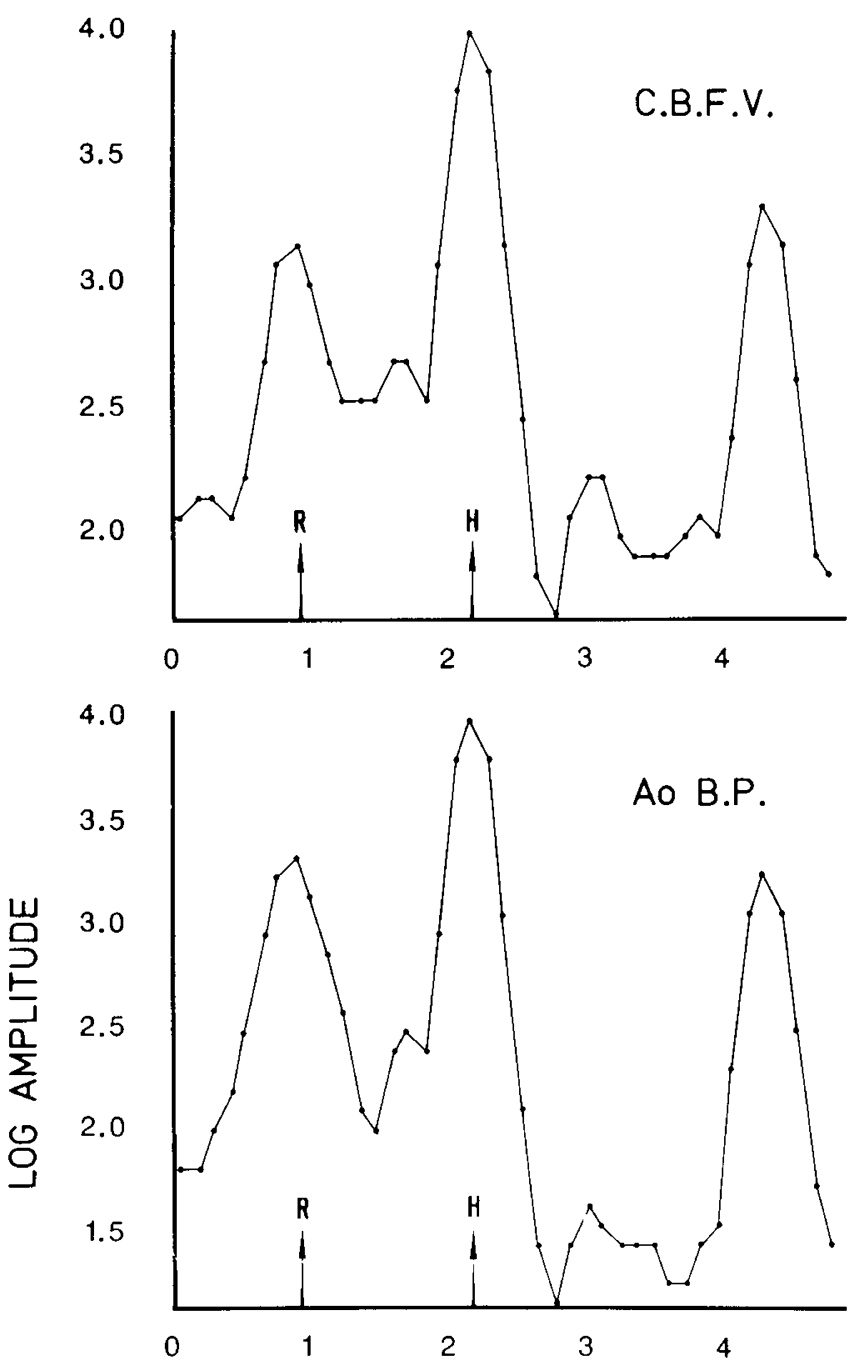

FREQUENCY ( $\mathrm{Hz})$

Fig. 2. Power spectra of BP (lower panel) and CBFV (upper panel) recorded simultaneously during spontaneous respiration. Note the transmission of the R component from BP to CBFV and the similarity of the two spectra obtained. 


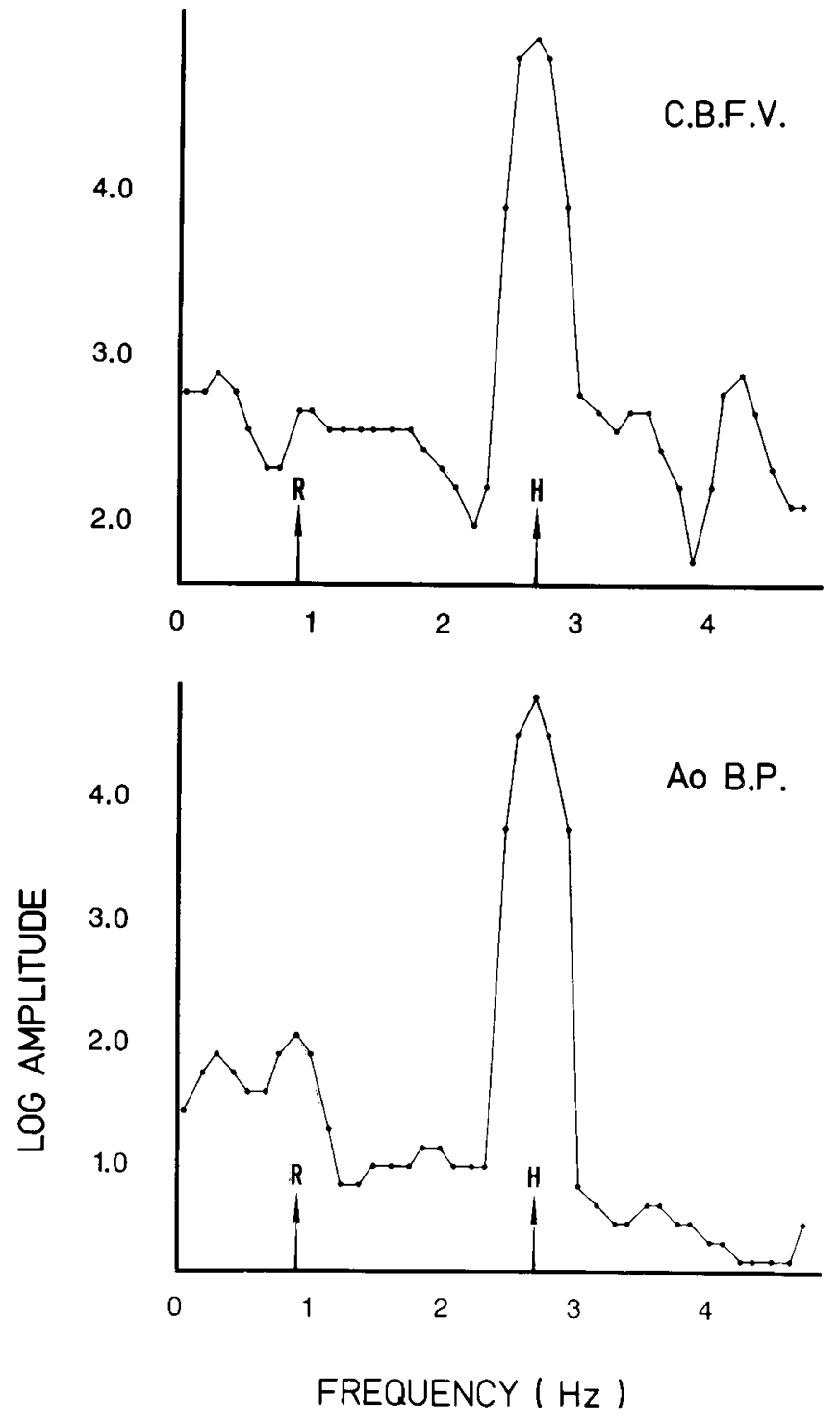

Fig. 3. Power spectra of $\mathrm{BP}$ and $\mathrm{CBFV}$ during paralysis and ventilation. The $\mathrm{H} / \mathrm{R}$ ratio in $\mathrm{BP}$ is greatly increased, indicating less respiratory interference in blood pressure than in Figure 2. Note that the R component in CBFV is barely visible.

sion coefficient of $-0.922(p=0.0001)$. There was better correlation between $\mathrm{cv} \%$ and $\mathrm{H} / \mathrm{R}$ ratios (regression coefficient = $-1.359, p<0.0001$ ) in spontaneously breathing infants than in paralyzed and ventilated infants (regression coefficient $=+0.184$, $p=0.55$ ). The $\mathrm{H} / \mathrm{R}$ ratio should be inversely correlated with the $\mathrm{cv} \%$ if both represent respiratory variation, but this was true only when infants were not paralyzed during mechanical ventilation. On 16 of 38 occasions infants who demonstrated a marked respiration-induced variation in BP had no clear respiratory component in CBFV (Fig. 4).

\section{DISCUSSION}

The origin of respiratory effects on $\mathrm{BP}$ and left ventricular function has been studied in the adult human (13) and animal models $(14,15)$. During spontaneous inspiration, left ventricular stroke volume falls, resulting in a fall in BP for those cardiac cycles. During expiration there is an increase in left ventricular stroke volume and BP rises slightly. Passive lung inflation during ventilation, however, results in an increase in left ventricular stroke volume, although this is less marked than the opposite effect of spontaneous inspiration. These observations were re-
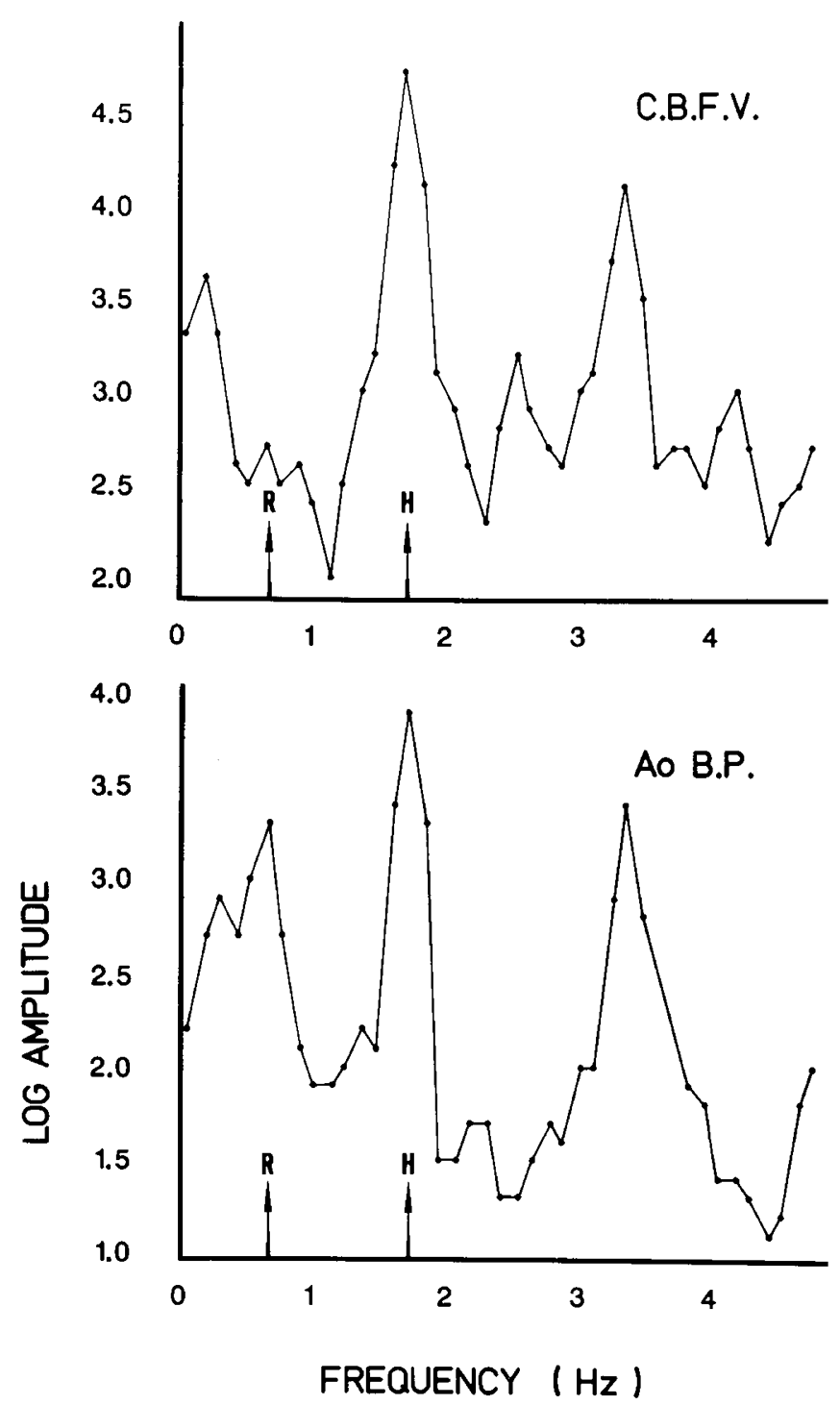

Fig. 4. Power spectra of BP and CBFV during spontaneous respiration. No R component is seen in CBFV although it is clearly seen in the $\mathrm{BP}$ spectrum. This pattern occurred in 16 of 38 simultaneous recordings.

cently repeated in the human newborn (16). Changes in several variables may affect left ventricular function during respiration, such as effective afterload, pulmonary venous return, and the transmitted effects of changes in right ventricular filling (17). The relative importance of these will vary during ventricular volume overload (18) and in the presence of shunts such as the patent foramen ovale (19).

Perlman et al. (1) have shown that instability of blood pressure in the premature infant with respiratory distress syndrome is readily transmitted to the cerebral circulation and carries a significantly increased risk of intraventricular hemorrhage. They and others have demonstrated the predominantly respiratory origin of these short-term fluctuations which are suppressed during muscle paralysis, a maneuver that was found to reduce the incidence and severity of subsequent intraventricular hemorrhage.

These and other studies have used a statistical index of variation (cv\%) to estimate the contribution of respiratory effort to cardiovascular instability, a measurement that is prone to error because it will include nonrespiratory interference and will be affected by changes in damping in the BP transduction system. By using spectral analysis of waveforms we have been able to quantify that component of variability specifically due to $R$ and, because the pulsatility of the waveform is the reference frequency 
component $(\mathrm{H})$, the effects of damping are eliminated. Dick et al. (20) have shown that two separately induced pressure waves at 2 and $3 \mathrm{~Hz}$, similar to those measured herein, are transmitted linearly in the aorta and proximal arterial system of the dog. We have shown that the $\mathrm{H}$ component is stable in 20 successive records during mechanical ventilation despite short-term fluctuations in mean aortic BP. The frequency of the R component will be fixed during regular respiration, or paralysis and mechanical ventilation and that of the $\mathrm{H}$ component will be fixed because of the lack of heart rate variability in the sick, premature infant (21).

Using similar methods of data collection to those of Perlman et al. (1) we have been able to identify components $H$ and $R$ even during paralysis. The new method can be used to follow changes in cardiovascular variability in response to therapeutic maneuvers and demonstrates the effect of withdrawing spontaneous respiratory effort during paralysis. The inability of the $\mathrm{cv} \%$ to differentiate pure respiratory effects on BP from those of motor activity may account for discrepancies between $H / R$ ratio and cv\% in the same recordings, although the overall correlation in the nonparalyzed group was good because periods of motor activity and irregular respiration were excluded. The degree of damping in the BP system we used may have suppressed the cv\% so much that it became unreliable at the low levels of variability encountered during paralysis when there was no correlation between the two measures. The dynamic characteristics of the system are nevertheless similar to many in clinical use where a limiting feature is the presence of a necessarily narrow-bore catheter. (The substitution of a 5-French gauge arterial catheter in our system caused a fall in the damping coefficient from 0.61 to 0.47 when measured in vitro by the rapid flush technique.)

Unlike Perlman et al. (1) we have not seen evidence of respiratory interference in all CBFV traces studied even when these were obvious in the aortic BP signal. The number of simultaneous $\mathrm{BP}$ and $\mathrm{CBFV}$ recordings studied is not yet great enough to allow us to determine the effects of gestational age, postnatal age, or illness on the degree of transmission of respiratory BP components into the cerebral circulation.

We believe that an estimation of the degree of transmission of a naturally occurring disturbance of BP to the cerebral vasculature may provide a means of assessing the effective tone of the intervening arteries and an insight into the regulation of brain blood flow in the first days of life.

Acknowledgments. The authors thank Dr. R. I. Kitney, Department of Electrical Engineering, Imperial College, and Mrs. Jane Wadsworth, Lecturer in Medical Statistics, St. Mary's Hospital Medical School, London for helpful advice.

\section{REFERENCES}

1. Perlman JM, McMenamin JB, Volpe JJ 1983 Fluctuating cerebral blood flow velocity in respiratory distress syndrome. N Engl J Med 309:204-209

2. Lou HC, Lassen NA, Friis-Hansen B 1979 Impaired autoregulation of cerebral blood flow in the distressed newborn infant. J Pediatr 94:118-121

3. Valimaki IAT, Korvenranta H, Kero PO 1984 Monitoring of an infant with RDS. In: Raivio KO, Hallman N, Kouvalainen K, Valimaki I (eds) Respiratory Distress Syndrome. Academic Press, London, pp 227-240

4. Perlman JM, Volpe JJ 1983 Suctioning in the preterm infant: effects on cerebral blood flow velocity, intracranial pressure, and arterial blood pressure. Pediatrics 72:329-334

5. Lou HC, Friis-Hansen B 1979 Arterial blood pressure elevations during motor activity and epileptic seizures in the newborn. Acta Pediatr Scand 68:803806

6. Girling DJ 1972 Changes in heart rate, blood pressure, and pulse pressure during apnoeic attacks in newborn babies. Arch Dis Child 47:405-410

7. Perlman JM, Goodman S, Kreusser KL, Volpe JJ 1985 Reduction in intraventricular hemorrhage by elimination of fluctuating cerebral blood-flow velocity in preterm infants with respiratory distress syndrome. $N$ Engl $J$ Med 312:1353-1357

8. South M, Morley CJ 1986 Synchronous mechanical ventilation of the neonate. Arch Dis Child 61:1190-1195

9. McDonald DA 1974 The numerical analysis of circulatory waveforms. In: McDonald DA (ed) Blood Flow in Arteries. Edward Arnold, London, pp 146-173

10. Kitney RI 1987 Beat-by-beat interrelationships between heart rate, blood pressure, and respiration. In: Kitney RI, Rompelman O (eds) The Beat-byBeat Investigation of Cardiovascular Function. Oxford University Press, Oxford, pp 146-178

11. Gardner RM 1981 Direct blood pressure measurement-dynamic response requirements. Anesthesiology 54:227-236

12. Armitage P 1974 Statistical Methods in Medical Research. Blackwell, London, pp 238-239

13. Perschau RA, Pepine CJ, Nichols WW, Downs JB 1979 Instantaneous blood flow responses to positive end-expiratory pressure with spontaneous ventilation. Circulation 59:1312-1318

14. Robotham JL, Lixfeld W, Holland L, MacGregor D, Bryan AC, Rabson J 1978 Effects of respiration on cardiac performance. J Appl Physiol 44:703709

15. Charlier AA 1967 Beat to beat hemodynamic effects of lung inflation and normal respiration in anesthetized and conscious dogs. Thesis, Editions Arscia, Brussels

16. Reller M, Kotagal UR, Meyer RA, Kaplan S 1986 Duration of ventricular ejection during spontaneous breathing and positive pressure ventilation in newborn infants. Biol Neonate 50:130-135

17. Permutt S, Wise RA 1986 Mechanical interaction of respiration and circulation. In: Handbook of Physiology III, Section 3. American Physiological Society, Washington, D.C., pp 647-656

18. Ditchey RV 1984 Volume-dependent effects of positive airway pressure on intracavitary left ventricular end-diastolic pressure. Circulation 69:815-821

19. Spritzer R, Versprille A 1987 The shunt through the foramen ovale during mechanical ventilation. Pediatr Res 21:388A(abstr)

20. Dick DE, Kendrick JE, Matson GL, Rideout VC 1968 Measurement of nonlinearity in the arterial system of the dog by a new method. Circ Res 22:101-111

21. Jenkins J, Mitchell H, McClure G, Reid M 1980 The measurement and analysis of neonatal heart rate and variability. In: Rolfe $P$ (ed) Fetal and Neonatal Physiological Measurements. Pitman Medical, London, pp 90 103 